\title{
Use of Bodipy-labeled sphingolipid and cholesterol analogs to examine membrane microdomains in cells
}

\author{
David L. Marks · Robert Bittman · Richard E. Pagano
}

Accepted: 9 September 2008 / Published online: 27 September 2008 (C) Springer-Verlag 2008

\begin{abstract}
Much evidence has accumulated to show that cellular membranes such as the plasma membrane, contain multiple "microdomains" of differing lipid and protein composition and function. These domains are sometimes enriched in cholesterol and sphingolipids and are believed to be important structures for the regulation of many biological and pathological processes. This review focuses on the use of fluorescent (Bodipy) labeled analogs of sphingolipids and cholesterol to study such domains. We discuss the similarities between the behavior of Bodipy-cholesterol and natural cholesterol in artificial bilayers and in cultured cells, and the use of Bodipy-sphingolipid analogs to visualize membrane domains in living cells based on the concentration-dependent monomer-excimer fluorescence properties of the Bodipy-fluorophore. The use of Bodipy-Derythro-lactosylceramide is highlighted for detection of domains on the plasma membrane and endosome membranes, and the importance of the sphingolipid stereochemistry in modulating domain formation is discussed. Finally, we suggest that Bodipy-sphingolipids may be useful in future studies to examine the relationship between
\end{abstract}

D. L. Marks · R. E. Pagano

Thoracic Diseases Research Unit, Department of Medicine, Mayo Clinic College of Medicine, Stabile 8, 200 First Street, SW, Rochester, MN 55905-0001, USA

D. L. Marks · R. E. Pagano $(\bowtie)$

Department of Biochemistry and Molecular Biology,

Mayo Clinic College of Medicine, Stabile 8, 200 First Street,

SW, Rochester, MN 55905-0001, USA

e-mail: pagano.richard@mayo.edu

R. Bittman

Department of Chemistry and Biochemistry, Queens College,

The City University of New York, Flushing, NY 11367, USA membrane domains at the cell surface and domains enriched in other lipids and proteins on the inner leaflet of the plasma membrane.

Keywords Lipid rafts · Gangliosides ·

Glycosphingolipids $\cdot$ Golgi apparatus $\cdot$ Sterols

\section{Introduction}

Microdomains represent local regions of membranes that have a different overall composition from the bulk membrane and are thought to act as organizing centers to sequester particular lipids and proteins. Although debate continues concerning various aspects of microdomains (e.g., size, number, lifetime), the concept has generated important new ideas about the roles of lipids and membranes in cellular functions (Kirkham and Parton 2005; Li et al. 2005; Rajendran and Simons 2005). Evidence for microdomains in various cellular membranes that differ in their properties from those of the bulk lipid bilayer membrane comes from multiple approaches including "detergent insolubility" of membrane components, cross-linking of membrane proteins and lipids, biophysical studies of constrained lateral diffusion in membranes, various fluorescence methods (e.g., fluorescence energy transfer; fluorescence correlation spectroscopy), atomic force microscopy, and EM studies [for reviews, see (Anderson and Jacobson 2002; Edidin 2003; Lawrence et al. 2003; London and Brown 2000; Marguet et al. 2006; Parton and Hancock 2004; Simons and Toomre 2000)]. One class of microdomains, sometimes referred to as "lipid rafts" (Pike 2006; Simons and Ikonen 1997), are regions of the membrane enriched in sphingolipids (SLs) and cholesterol (See Sect. "Labeling of microdomains in living cells"). In model 
membranes prepared with mixtures of phospholipids and cholesterol, microdomains with a short-range order (liquidordered or $1_{0}$ domains) have been observed by fluorescence imaging to coexist with a disordered liquid phase $\left(l_{d}\right)$. These in vitro liquid-ordered domains have some properties in common with cholesterol/SL-enriched microdomains that exist in cell membranes in vivo. However, microdomains of cell membranes are infinitely more complex in terms of lipid composition, the presence of proteins that may modulate microdomain structure, and the likelihood that multiple types of microdomains may be present in the same membrane. An understanding of cholesterol-SL microdomains remains a challenge, and progress in this field is dependent on the development of spectroscopically observable lipid analogs that faithfully reflect the properties of their parent molecule. This review focuses on the use of SL and cholesterol analogs labeled with the Bodipy fluorophore to study microdomains in cell membranes in the membranes of living cells.

\section{Bodipy-lipid analogs: chemistry; spectral and physical properties}

\section{Spectral Properties of Bodipy fluorophores}

The fluorophore known as Bodipy [boron dipyrromethene (4,4-difluoro-5,7-dimethyl-4-bora-3a,4a-diaza-s-indacene); see Fig. 1a] was originally used by us in studies of SL synthesis and transport along the secretory pathway in cultured mammalian cells (Pagano et al. 1991). It is now called "Bodipy-FL" by Invitrogen but is referred to as "Bodipy" throughout this review. Because of its unique spectral properties and superior photo-stability compared to other fluorophores such as nitrobenz-2-oxa-1,3-diazol-4-yl (NBD) and fluorescein, we subsequently generated other Bodipy-analogs for lipid trafficking studies. Lipid analogs labeled with the Bodipy fluorophore exhibit monomer/excimer fluorescence emission. Thus, when present in membranes at relatively low concentrations and excited with blue light (e.g., $470 \mathrm{~nm}$ ) the probe emits at green wavelengths (peak at $515 \mathrm{~nm}$ ); however, when the fluorescent probe is present at higher concentrations, the green emission is partially quenched and a second peak of fluorescence is observed at red wavelengths $(\sim 620 \mathrm{~nm})$. This concentration-dependent spectral shift occurs in vitro [e.g., with Bodipy-ceramide (Cer) in liposomes; Fig. 1b] and in living cells [e.g., using Bodipy-lactosylceramide (LacCer); Fig. 1c], thus allowing one to distinguish regions of a membrane that contain relatively high concentrations of the lipid analog (and fluorescent red) from other regions in which the probe is present at lower concentrations that fluorescent green. Another example of this phenomenon is shown in
Fig. 1d in which Bodipy-Cer and its metabolites accumulate in the Golgi apparatus of human skin fibroblasts. The apparent molar density of the Bodipy-analog can be estimated in the region of interest by quantifying the intensities of the red and green emissions and comparing this to a calibration curve relating red/green emission ratios to the molar density of the Bodipy-lipid analog in liposomes imaged under the same conditions as the cells (Chen et al. 1997; Pagano et al. 1991).

A number of modifications to the Bodipy group have been prepared by Invitrogen and others ( $\mathrm{Li}$ and Bittman 2007; Qin et al. 2007). These substitutions to the fluorophore result in analogs with different spectral properties. Of these novel fluorophores, only Bodipy-TR, which has fluorescence properties similar to Texas Red, has been used to label SL analogs. Several studies have used Bodipy-TR-Cer as a probe for the Golgi apparatus (Field et al. 2000; Gangalum et al. 2004).

\section{Structures of SLs and analogs}

Sphingolipids are a large class of lipids found in eukaryotes as well as in some bacteria. In addition to their functions within cholesterol-enriched microdomains, SLs play important roles in cell signaling, cell-cell interactions, and pathophysiological processes (Ariga et al. 2008; Hannun and Obeid 2008; Holland and Summers 2008; Regina Todeschini and Hakomori 2008). In mammalian cells there are hundreds of molecular species of SLs with sometimes confusing trivial and systematic names [for comprehesive reviews, see (Fahy et al. 2005; Hakomori 2003; Shayman and Radin 1991)]. However, the basic structure of SLs can be considered to be a sphingosine core (also known as the "long-chain base"), an amidelinked fatty acid, and a polar head group (see Fig. 2a). Cer, from which sphingomyelin (SM) and glycosphingolipids (GSLs) are derived, lacks the phosphocholine and carbohydrate head group present in SM and GSLs, respectively. Most cell types synthesize glucosylceramide (GlcCer), which consists of glucose in a $\beta$-anomeric linkage with Cer. Other sugars are added to the glucose head group to form higher-order GSLs (Fig. 2a). For example, incorporation of galactose (Gal) into GlcCer forms the disaccharyl-SL, LacCer (Fig. 2b). GSLs that possess sialic acid residues are called gangliosides (e.g., see $\mathrm{GM}_{1}$ in Fig. 2a). In some tissues, $\beta$-linked galactosyl-Cer (GalCer) is synthesized, which can be further modified by a sulfate ester to form sulfatide (Fig. 2a). Differences in fatty acid chain length, variation in saccharide chains, and variations in sphingosine chain length and structure (e.g., hydroxyl groups, double bonds) account for the great numbers of individual SL molecular species found (Bittman 2008). 
Fig. 1 Spectral characteristics of the Bodipy fluorophore. a Chemical structure of the unsubstituted core of the Bodipy fluorophore. b Fluorescence emission spectral of lipid vesicles containing various amounts of Bodipy- $\mathrm{C}_{5}$-ceramide (Bodipy-Cer). Small unilamellar vesicles were formed from POPC and 2, 5, 10, 20, and $50 \mathrm{~mol} \%$ Bodipy-Cer (total lipid content was $31 \mu \mathrm{M})$. Excitation was at $480 \mathrm{~nm}$. Inset: Ratio of relative fluorescence intesities at 620 and $515 \mathrm{~nm}$ vs mol \% Cer. [Adapted from (c) Pagano et al. 1991. Originally published in The Journal of Cell Biology 113:1267-1279]. c Human skin fibroblasts labeled for $30 \mathrm{~min}$ at $10^{\circ} \mathrm{C}$ with $2.5 \mu \mathrm{M}$ Bodipy- $\mathrm{C}_{5^{-}}$ lactosylceramide (Bodipy-LacCer) followed by $5 \mathrm{~min}$ at $37^{\circ} \mathrm{C}$. Note the heterogeneous labeling of endocytic vesicles at red and green wavelengths, indicating respectively high and low concentrations of Bodipy-LacCer in the endosomal membranes. d) Human skin fibroblasts labeled with Bodipy-Cer for $30 \mathrm{~min}$ at $10^{\circ} \mathrm{C}$. Note Golgi labeling in red and more diffuse labeling of intracellular membranes in green. For $\mathbf{c}$ and $\mathbf{d}$, samples were excited with blue light (470 $\mathrm{nm}$ ) and emission was visualized with green $(540 \mathrm{~nm})$ and red ( $\geq 590 \mathrm{~nm}$ ) filters. Bar $=1 \mu \mathrm{m}$

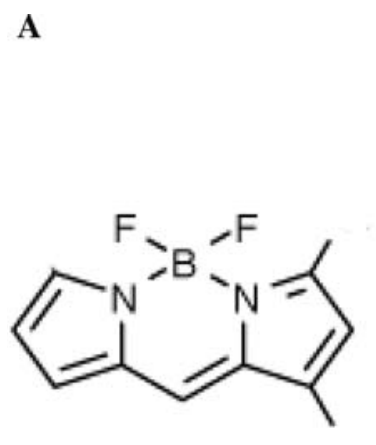

$B$

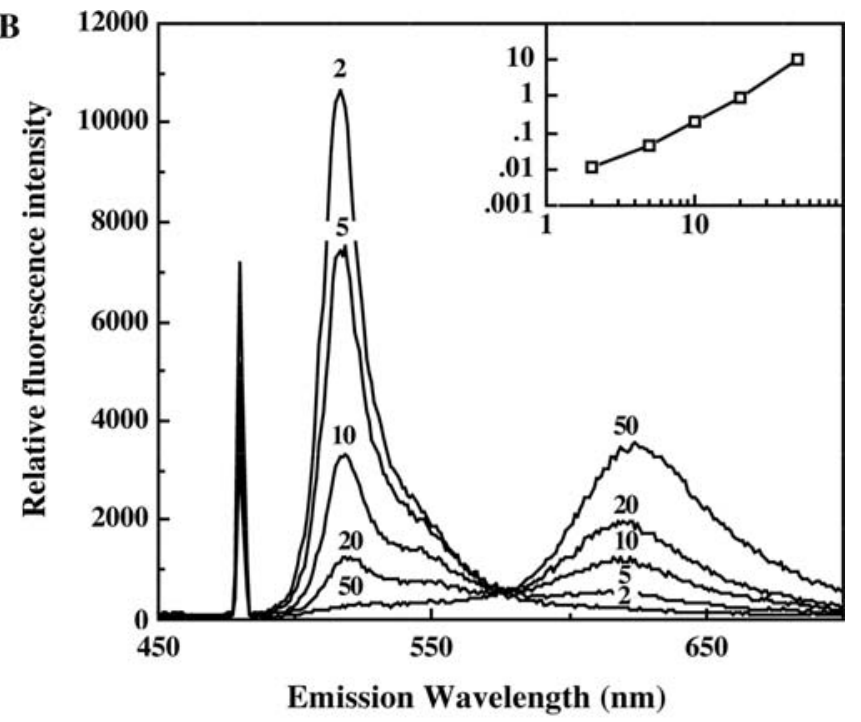

C BODIPY-Green
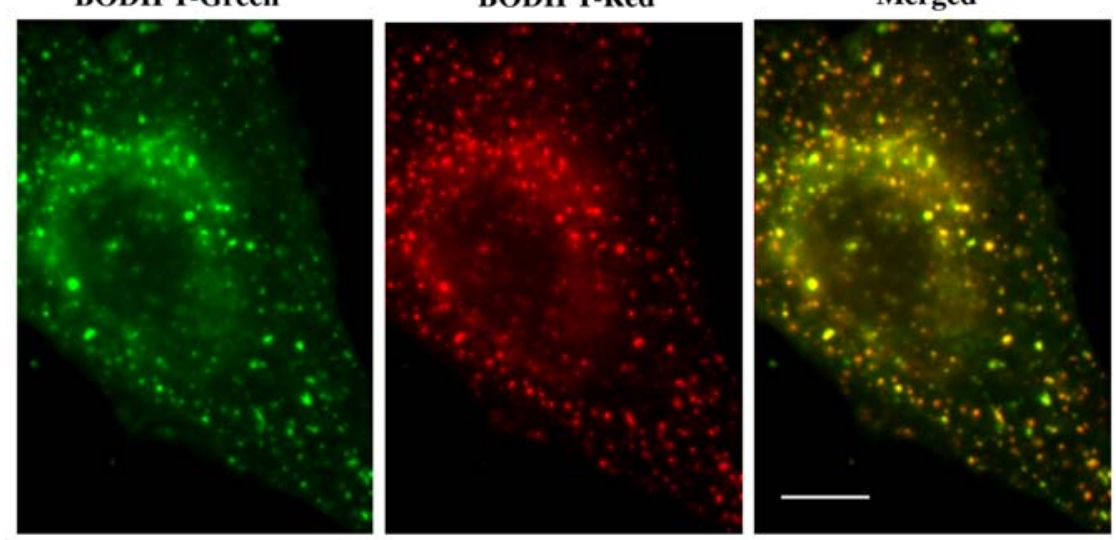

D
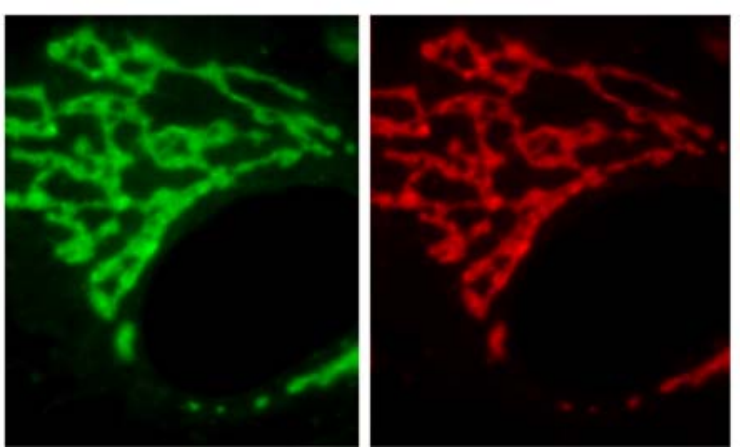

\section{Unusual SLs}

Carbons 2 and 3 of the sphingosine backbone are asymmetric; thus, four potential stereoisomers of SLs can be envisioned (see Fig. 2b for an example showing the stereoisomers of LacCer). All natural mammalian SLs have the D-erythro (or $2 S, 3 R$ ) stereochemistry. As noted below, alterations in the stereochemistry of the long-chain base drastically changes the biophysical and biological behavior of LacCer. Interestingly, SLs with different (L-threo or $2 \mathrm{~S}, 3 \mathrm{~S})$ stereochemistry occur in plants, marine invertebrates, and fungi (Emura et al. 2005; Gao et al. 2004; Inagaki et al. 2006; Ishii et al. 2006; Ling et al. 2006; Oueslati et al. 2005; Yamada et al. 2000; Zhang et al. 2007). While all mammalian GSLs possess the $\beta$-anomeric linkage between the first sugar and Cer, an unusual set of $\alpha$-linked 
A

\section{Sphingolipid}

Cer

SM

GlcCer

GalCer sulfatide

\section{R-group}

$\mathrm{H}-$
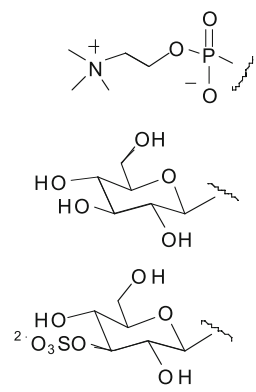

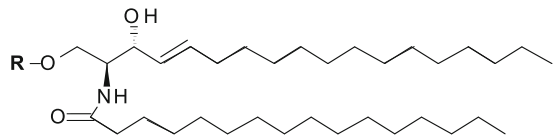

N palmitoyl Sphingolipid

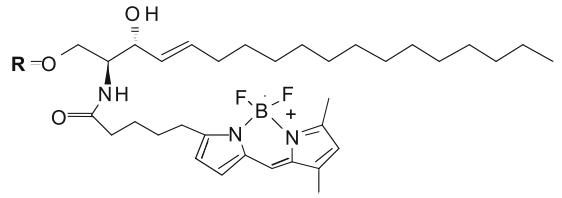

BODIPY C5 Sphingolipid

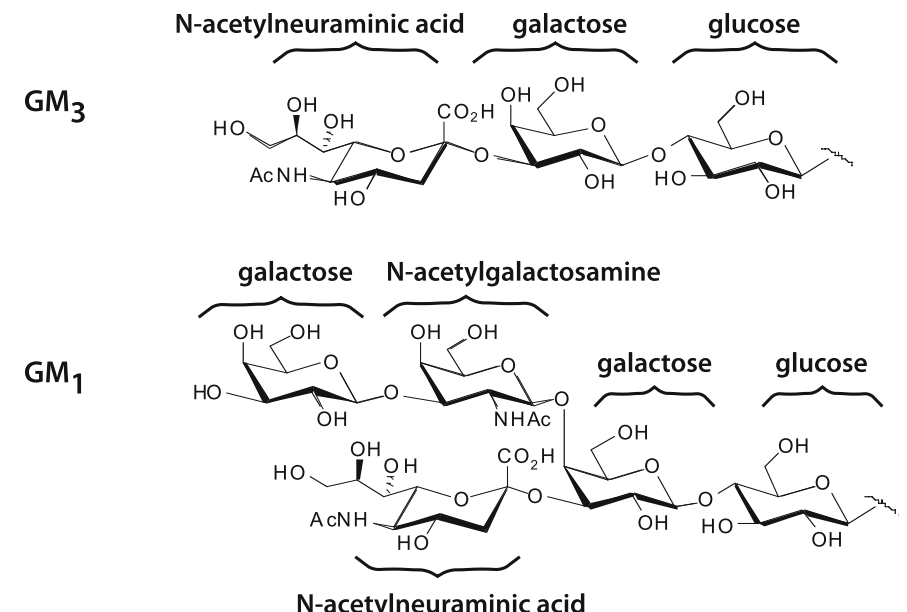

$\mathrm{N}$-acetylneuraminic acid

B

2S, 3R (D-erythro)

$2 \mathrm{R}, 3 \mathrm{~S}$ (L-erythro)

2R, 3R (D-threo)

2S, 3S (L-threo)

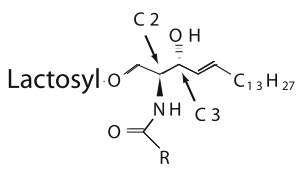

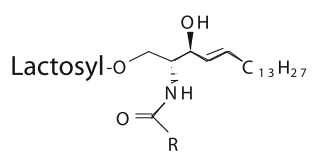

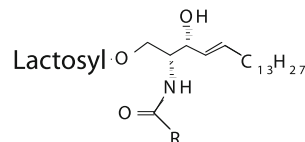<smiles>CC(=O)N[C@@H](COC(=O)OC(C)(C)C)[C@H](O)CCCN</smiles>

Lactosyl $=\underbrace{\mathrm{HOH}_{\mathrm{O}}}_{\mathrm{OH}}$

C

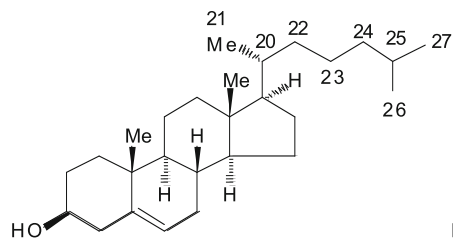

Cholesterol

Fig. 2 Structures of sphingolipids and Bodipy-cholesterol. a Model showing component parts of sphingolipids; sphingosine core, head groups, and fatty acid. In Bodipy-SLs the natural fatty acid is replaced by a Bodipy fatty acid. $R$ indicates head groups such as phosphocholine

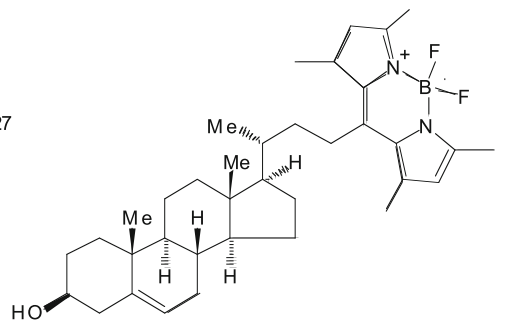

BODIPY Cholesterol

in SM and saccharides found in GSLs. b Illustration of four stereoisomers of LacCer indicating the asymmetric carbon atoms, C-2 and C-3 c Structures of cholesterol and Bodipy-cholesterol 
GSLs from microbes and marine sponges have been found to activate natural killer $\mathrm{T}$ cells. The reader is referred to (Bittman 2008) for a discussion of these and other unusual SLs.

\section{Bodipy-SL analogs}

We have prepared a variety of SL analogs in which the fatty acid moiety is replaced by a Bodipy fatty acid. These include Bodipy-Cer, -GlcCer, -GalCer, Maltosyl-Cer, -LacCer, -SM, $-\mathrm{GM}_{1}$, $-\mathrm{GM}_{3}$, and -sulfatide [see Fig. 2a and (Martin and Pagano 1994; Pagano et al. 1991; Singh et al. 2003; Watanabe et al. 1999)]. Some of these compounds are now available from Invitrogen. In most cases, the SL analogs were generated using Bodipy-labeled pentanoic acid (C5) as in Fig. 2a, although longer and shorter chain length Bodipy-fatty acids are commercially available and can be used, depending on the application. Unlike most fluorophores, Bodipy is relatively nonpolar and electrically neutral. These features make it desirable for the synthesis of SL probes since it replaces the natural fatty acid and, in principle, is capable of intercalating into membrane bilayers more readily than hydrophilic fluorophores. For example, in a comparison of the properties of Bodipy- vs NBD-labeled Cer analogs, it was found that the Bodipy fluorophore appears to better intercalate into the membrane bilayer than the NBD fluorophore (Chattopadhyay and London 1987; Kaiser and London 1998). A useful property of Bodipy-SLs, which is also true of NBD-SLs, is that they can be easily combined in a complex with defatted bovine serum albumin (DF-BSA) to make a water-soluble delivery system for transfer of the lipid analog to cells (Martin and Pagano 1994). In addition, Bodipy-SLs in the outer leaflet of the PM of cells can easily be removed by adding DFBSA to the medium in a process called "back exchange," thus allowing the visualization of fluorescent lipid which has been internalized by the cell without interference from fluorescent lipid remaining at the PM (Koval and Pagano 1989; Martin and Pagano 1987, 1994).

\section{Cholesterol and Bodipy-cholesterol analogs}

Cholesterol is a key lipid component of mammalian cell membranes where it plays a unique role in determining membrane properties [for recent reviews, see (Ikonen 2008; Maxfield and Tabas 2005; Schroeder et al. 2001; Simons and Ehehalt 2002)]. For example, cholesterol affects membrane fluidity, rigidity, and as mentioned above, the ability of membranes to form laterally segregated microdomains. To further understand cholesterol function and transport in the living cell, fluorescently labeled cholesterol analogs would be very useful. An ideal cholesterol probe would exhibit properties similar to those of cholesterol in the highly packed and ordered environment typical of lipid rafts, would not markedly perturb membrane structure, and would possess photo-physical properties suitable for use in live cell fluorescence microscopy of cholesterol-rich membrane domains. The development of such an analog has been problematic, because the size, hydrophobicity, and the site of attachment of the fluorophore to the sterol molecule all can affect how the probe molecule is partitioned in membrane domains. Several analogs of cholesterol were synthesized in which Bodipy has been inserted into the aliphatic tail of the free sterol. One compound, referred to here as Bodipy-cholesterol (Fig. 2c) in which carbon-24 of cholesterol is linked directly to a Bodipy moiety, exhibited similar physical behavior to cholesterol ( $\mathrm{Li}$ and Bittman 2007; $\mathrm{Li}$ et al. 2006; Shaw et al. 2006). In particular, in model membranes Bodipy-cholesterol partitioned into liquid-ordered domains, as judged by (a) its distribution into a Triton X100 low-density insoluble fraction (a biochemical method based on the resistance of lipid rafts to be extracted by a detergent) and (b) atomic force microscopy-confocal fluorescence imaging data. Furthermore, the rate of desorption of Bodipy-cholesterol from monolayers was similar to that of cholesterol on addition of a water-soluble cyclodextrin to mixed monolayers with SM. Bodipy-cholesterol also preferentially partitioned into the $1_{0}$ phase of giant unilamellar vesicles, in contrast to the $1_{\mathrm{d}^{-}}$-domain specificity of the fluorescent probe 1,1'-didodecyl-3,3,3',3'-tetramethylindocarbocyanine perchlorate $\left(\right.$ diI- $\left._{12}\right)$ (Florly S. Ariola, Robert Bittman, and Ahmed A. Heikal, unpublished observations). These data demonstrate the potential of this analog for studying lipid-lipid interactions, lipid order, and membrane fluidity of biologically relevant $l_{\mathrm{o}}$ domains.

\section{Labeling of microdomains in living cells}

\section{Definitions of "microdomains"}

The concept of lateral heterogeneity of biological membranes has been discussed for over two decades. Much evidence has been accumulated to show that membranes [e.g., the plasma membrane (PM)] consist of multiple domains (or microdomains) of differing lipid and protein composition and function (Mishra and Joshi 2007). In this review we focus on SL- and cholesterol-enriched microdomains, sometimes referred to as "rafts," detergent-resistant membranes, or GSL-enriched membranes. Even within this category, it is expected that there exist multiple types of microdomains with different functions. For example, one category of SL- and cholesterol-enriched microdomain is caveolae,: flask-shaped 50-100 nm invaginations of the PM defined by the presence of the caveolin protein (Jacobson et al. 2007). Apparently distinct from caveolae are 
microdomains enriched in glycosylphosphatidyl-inositol (GPI)-anchored proteins (Schnitzer et al. 1995). In a further example of multiple microdomains within the same cell type, Hakomori and colleagues used detergent insolubility fractionation of B16 melanoma cells combined with immuno-isolation to separate $\mathrm{a} \mathrm{GM}_{3}$ ganglioside-enriched fraction that also contained SM and RhoA from a caveolinenriched fraction that did not contain $\mathrm{GM}_{3}$ (Iwabuchi et al. 1998).

The size of putative microdomains has been explored, and estimates from $5 \mathrm{~nm}$ to $1 \mu \mathrm{m}$ have been reported using a variety of technologies (Harding and Hancock 2008; Jacobson et al. 2007). Current consensus is that microdomains are typically smaller than 200-300 nm (Devaux and Morris 2004; Jacobson et al. 2007; Mishra and Joshi 2007), but this size is too small to be visualized by conventional fluorescence microscopy. However, microdomains can be induced to coalesce into larger structures by certain stimuli (Harder and Engelhardt 2004; Kusumi and Suzuki 2005). For example, cross-linking of the GPI-anchored protein placental alkaline phosphatase with antibodies causes it to move into patches which also contain $\mathrm{GM}_{1}$ ganglioside and the src-like protein tyrosine kinase, fyn (Harder et al. 1998).

\section{Cell fractionation studies}

\section{Bodipy-LacCer}

Biochemical studies were performed to determine if Bodipy-LacCer partitioned into cellular microdomains. Bodipy-LacCer $(5 \mu \mathrm{M})$ was incubated with human skin fibroblasts for $30 \mathrm{~min}$ at $10^{\circ} \mathrm{C}$. Cells were then washed and then fractionated using the detergent-free method for isolating microdomains developed by Smart et al. (1995). In this method, an Optiprep gradient is used to separate the buoyant microdomain fraction from other membranes. As shown in Fig. 3a, Cav1, a marker for microdomains, was distributed mainly near the top of the gradient. Clathrin heavy chain (Fig. 3a) and the bulk of cell protein (not shown) were distributed at the bottom of the gradient. Bodipy-LacCer was quantified in gradient fractions and found to occur mainly in the upper fractions with Cav1 (Fig. 3b). Finally, when non-fluorescent C8-LacCer (which stimulates the coalescence of microdomains; see below) was added to cells with Bodipy-LacCer, an even greater portion of Bodipy-LacCer was recovered in the Cav1-enriched fractions. However, when Bodipy-LacCer was added after cells were homogenized (post-lysis control), the fluorescent probe was distributed mainly in lower fractions (Fig. 3b). This control shows that the distribution of Bodipy-LacCer in the buoyant microdomain fractions occurs in the living cells and is not due to the scrambling of the lipid after
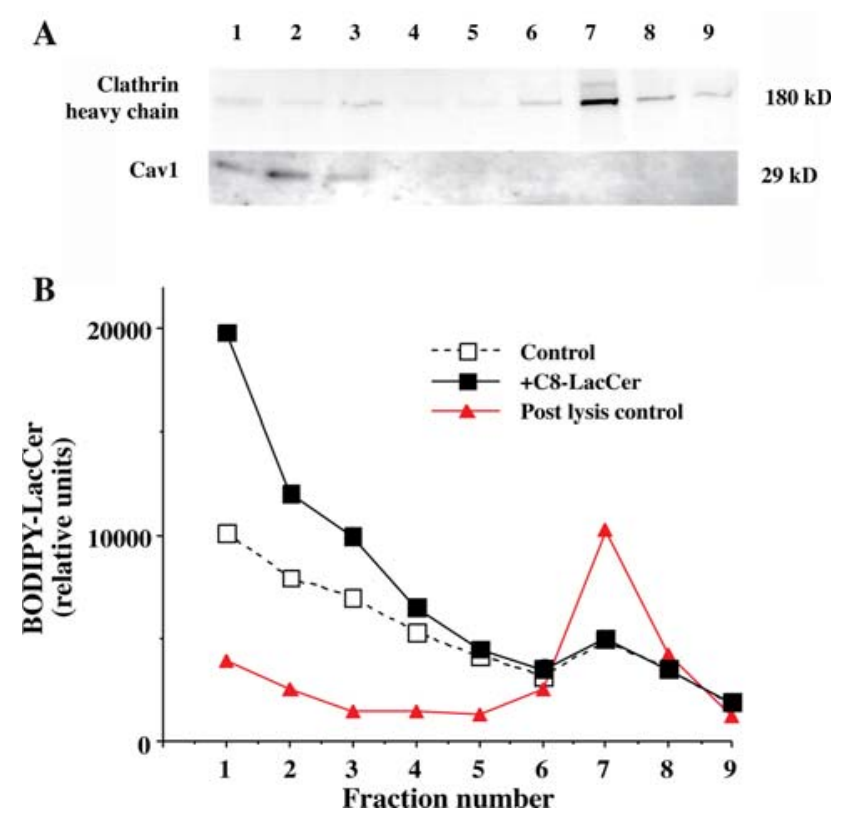

Fig. 3 Behavior of Bodipy-LacCer in cell fractionation studies. a, b Human skin fibroblasts were incubated with $5 \mu \mathrm{M}$ Bodipy-LacCer in HMEM for $30 \mathrm{~min}$ at $10^{\circ} \mathrm{C}$ and then washed. Cells were then scraped and fractionated by the detergent-free method of Smart et al (1995) to recover buoyant membrane microdomains. Data shown are from the last step of the fractionation using an Optiprep gradient. Western blotting a shows that Cav1, a marker for microdomains, occurs in early, buoyant fractions (1-3) whereas clathrin heavy chain as well as the bulk of protein (not shown) occur in the later fractions (6-9). Equal volumes were loaded for western blots. b The distribution of BodipyLacCer was examined by lipid extraction, thin layer chromatography, and image analysis, and shows that the fluorescent lipid is distributed mainly in the buoyant fractions

homogenization. In contrast, when Bodipy-LacCer was added to cells, and cells were subjected to cold $1 \%$ Triton $\mathrm{X}-100$ treatment and sucrose gradient fractionation, Bodipy-LacCer was found associated with the Triton X100 soluble fraction and not with the insoluble fraction (unpublished data). Thus, the ability of Bodipy-LacCer to partition into domains in living cells does not correlate with its Triton X-100 solubility.

\section{Bodipy-cholesterol and radiolabeled cholesterol}

Overnight incubation of Chinese hamster ovary (CHO) cells with Bodipy-cholesterol in a medium containing lipoprotein-deficient serum resulted in bright labeling of cells with a negligible $(\sim 1 \%)$ increase in total cellular sterols. To compare the distribution of Bodipy-cholesterol with radiolabeled cholesterol in subcellular membranes, the cells were incubated overnight with both Bodipy-cholesterol and $\left[{ }^{3} \mathrm{H}\right]$ cholesterol, and the membranes were fractionated in the post-nuclear supernatant by sucrose density gradient centrifugation. Bodipy-cholesterol partitioned into cellular membranes with different equilibrium densities 
very similarly to $\left[{ }^{3} \mathrm{H}\right]$ cholesterol. To analyze if Bodipycholesterol exhibits affinity for DRMs, CHO cells labeled with Bodipy- and radiolabeled cholesterol were lysed in cold $1 \%$ Triton X-100, and the detergent-insoluble material was pelleted. The results indicate that Bodipy-cholesterol associated with DRMs to a similar extent as radiolabeled cholesterol (Hölttä-Vuori et al. 2008).

Fluorescence microscopy studies

\section{Visualization of PM microdomains with Bodipy-D-erythro-LacCer}

Our initial studies using Bodipy-D-erythro-LacCer were focused on its mechanism of endocytosis and subsequent cellular itinerary (Choudhury et al. 2002a; Martin and Pagano 1994; Puri et al. 1999; Puri et al. 2001; Sharma et al. 2003). We found that this lipid analog underwent endocytosis selectively via caveolae in multiple cell types [for a review of techniques to study mechanisms of endocytosis, see (Marks et al. 2005; Singh et al. 2007b)]. In these studies, 1-2 $\mu \mathrm{M}$ Bodipy-D-erythro-LacCer was typically incubated with cells at $10^{\circ} \mathrm{C}$ to allow binding to the PM and then warmed to $37^{\circ} \mathrm{C}$ to initiate endocytosis. When cells were viewed after the low temperature binding step but without warm up, Bodipy-D-erythro-LacCer exhibited a relatively homogeneous distribution on the PM (see Fig. 4a, top left panel). We subsequently noted that under certain conditions, Bodipy-D-erythro-LacCer could be induced to redistribute into non-homogeneous $\sim 1 \mu \mathrm{m}$ size patches with increased red fluorescence (indicating higher local fluorescent lipid concentrations) on the PM (Fig. 4a, middle and right panels). These conditions included (1) co-incubation with $20 \mu \mathrm{M}$ non-fluorescent C8-D-erythro-LacCer or with natural chain length GSLs, (2) use of higher concentrations $(2.5-10 \mu \mathrm{M})$ of Bodipy-D-erythro-LacCer (see Fig. 4a top middle and right panels), (3) co-incubation with cross-linking $\beta 1$-integrin antibody, and (4) co-incubation with cholera toxin B subunit (CTxB), plus anti CTxB antibody (Sharma et al. 2005; Singh et al. 2007a; Singh et al. 2006).

These red-orange patches of Bodipy-D-erythro-LacCer (appear yellow when red and green images are merged) have the expected characteristics of PM microdomains. (1) They are inhibited by treatment of cells with methyl- $\beta$ cyclodextrin, which reduces the cholesterol content of cells (Sharma et al. 2005). (2) These structures co-localize with CTxB bound to cell surface $\mathrm{GM}_{1}$ ganglioside, demonstrating that the microdomains are enriched in endogenous GSLs (Fig. 5a). Bodipy-D-erythro-LacCer domains also co-localize with PM $\beta 1$-integrin (Sharma et al. 2005) as shown in Fig. 4b. Since it is known that Bodipy-D-erythro-LacCer, $\beta 1$-integrin, and $\mathrm{CTxB}$ (at least in part) are internalized via caveolae, our results suggested that these microdomains consist of lipids and proteins that are poised for endocytosis via caveolae.

\section{Bodipy-LacCer stereoisomers}

We have also investigated the properties of the non-natural stereoisomer Bodipy-L-threo-LacCer, which differs from Bodipy-D-erythro-LacCer by having a different stereochemical configuration at only one carbon ( $\mathrm{C} 3$ of the longchain base; see Fig 2b). Unlike the D-erythro stereoisomer, Bodipy-L-threo-LacCer does not exhibit red-shifted PM domains at higher concentrations (Fig. 4a, lower panels) and does not co-localize with $\beta 1$-integrin (Fig. $4 \mathrm{~b}$, lower panels) at the PM. Interestingly, Bodipy-L-threo-LacCer also is not internalized selectively by caveolae but instead undergoes endocytosis by multiple routes (Singh et al. 2006). These studies suggest that Bodipy-L-threo-LacCer does not concentrate in cholesterol and SL-enriched microdomains at the PM. Further studies have suggested a possible mechanism for the behavior of Bodipy-L-threo-LacCer. First, the fluorescence behavior of Bodipy-L-threo-LacCer in liposomes was compared to that of the D-erythro analog. Although Bodipy-D-erythro-LacCer in liposomes exhibits a red shift at higher concentrations, this red shift is not seen for the L-threo isomer in HSFs (Fig. 4c). A modeling study of the structures for Bodipy-D-erythro and -L-threo-LacCer suggested that in the L-threo stereoisomer the carbohydrate head group and the Bodipy-fatty acid are likely to be extended perpendicular from the sphingosine hydrocarbon chain, while in the D-erythro form the head group extends up and the Bodipy-fatty acid down in a more typical amphipathic lipid orientation (Fig. 4d). These results suggest that unlike the natural Bodipy-D-erythro-LacCer stereoisomer, the LacCer analog with the L-threo stereochemistry may be unable to associate closely with itself or with other GSLs, leading to an inability to incorporate into cholesterol- and SL-enriched domains. Additional studies with the other two unnatural stereoisomers of Bodipy-LacCer (D-threo and Lerythro) led to the conclusion that the stereochemistry at carbon 3 (see Fig. 2b) of the long-chain base is a key determinant for the inclusion of LacCer in SL/cholesterolenriched microdomains and for internalization via caveolae (Singh et al. 2006).

Studies with the fluorescent stereoisomers of LacCer prompted us to compare the properties of D-erythro and $\mathrm{L}$ threo stereoisomers of a non-fluorescent short-chain (octanoyl) analog of LacCer, C8-LacCer. We found that whereas D-erythro-C8-LacCer induced the coalescence of microdomains as detected by Bodipy-D-erythro-LacCer, CTxB, $\mathrm{BC}-\Theta$, and $\beta 1$-integrin Fab staining, as well as stimulating caveolar endocytosis (Sharma et al. 2005, Singh et al. 2007a), L-threo-C8-LacCer inhibited the induction of 

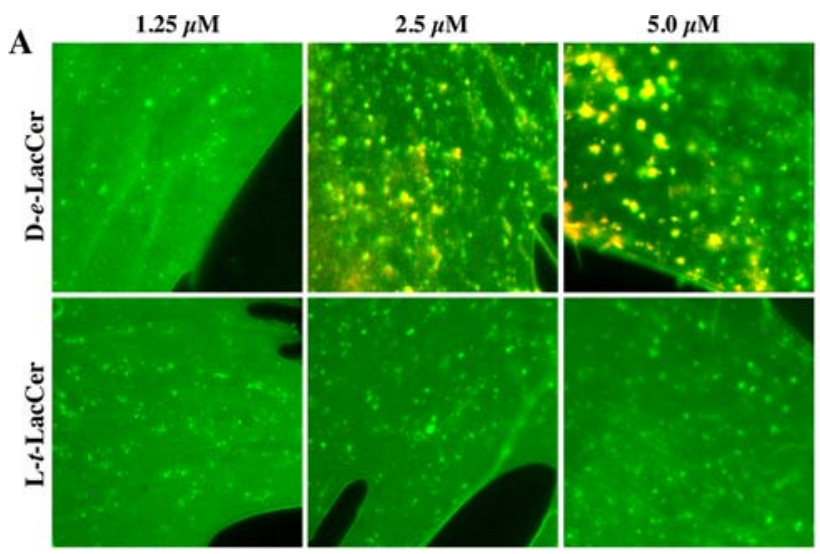

\section{D}

Green + Red - LacCer

B Blue - B1-integrin Fab

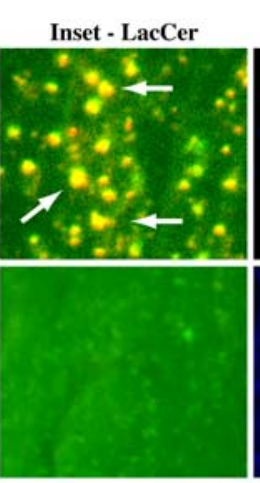

Inset - B1-integrin Fab
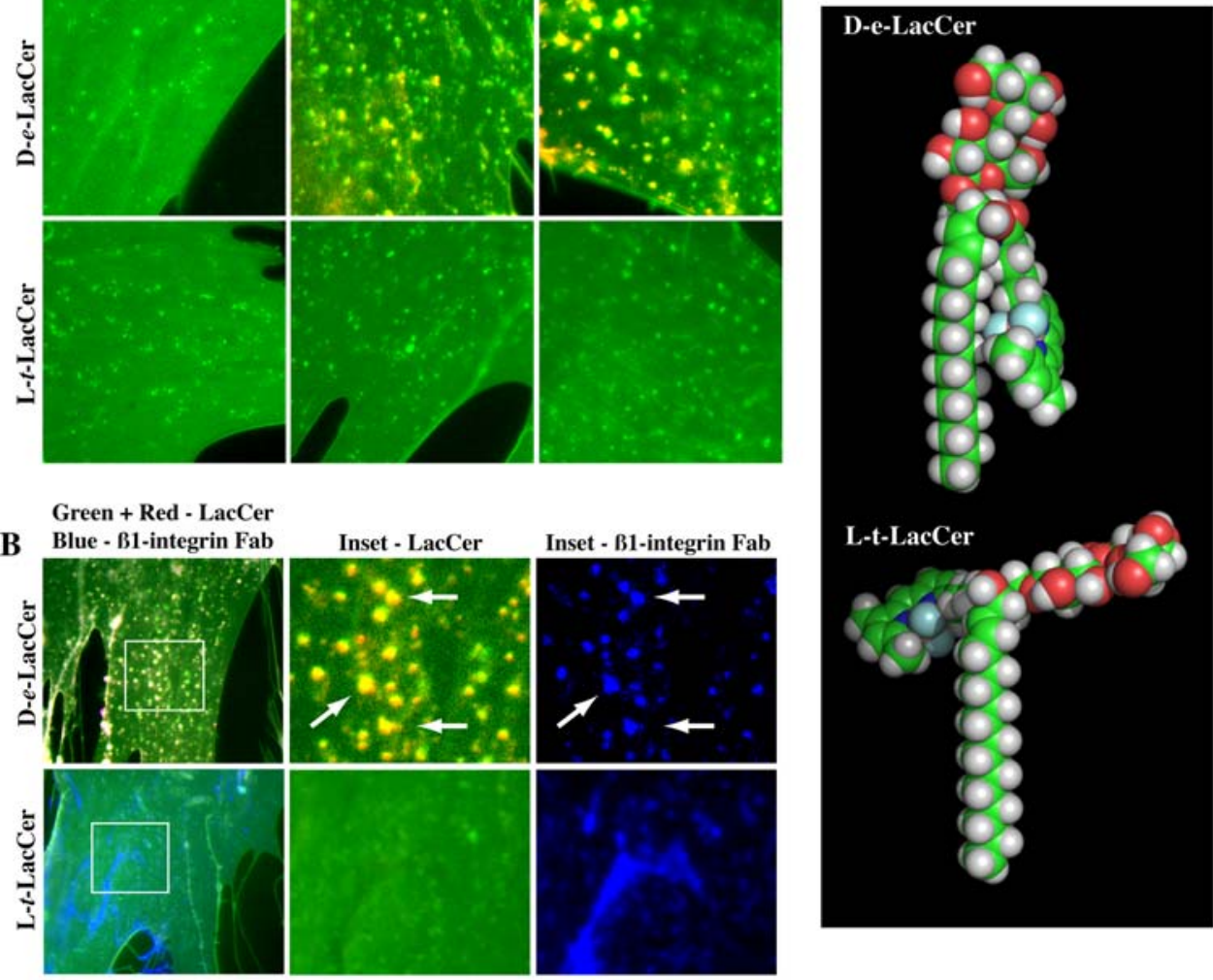

C
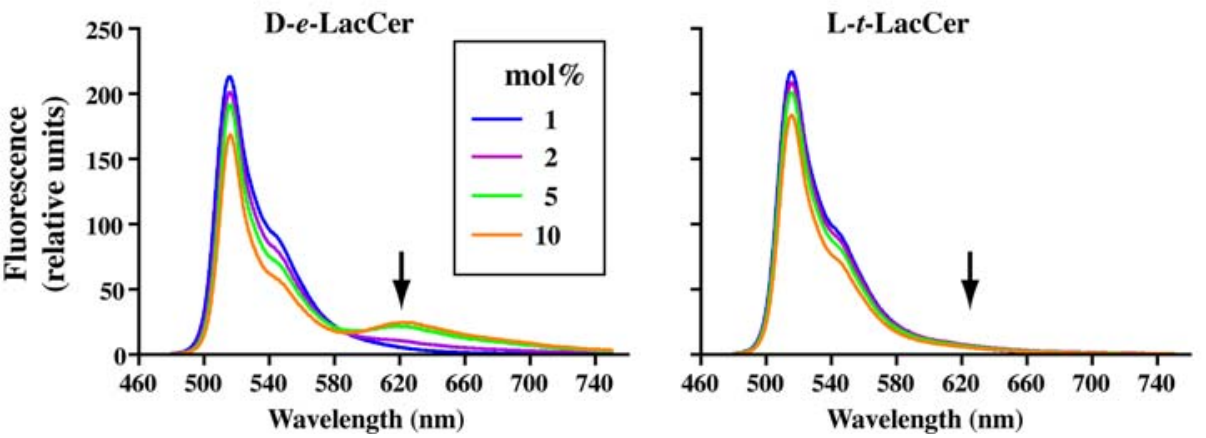

Fig. 4 Comparison of D-erythro and L-threo stereoisomers of BodipyLacCer. (a) Human skin fibroblasts were incubated with the indicated concentration of Bodipy-D-erythro (D-e) or L-threo- (L-t) LacCer for $30 \mathrm{~min}$ at $10^{\circ} \mathrm{C}$, washed, and observed under the fluorescence microscope at low temperature to inhibit endocytosis. Images were acquired simultaneously at green and red wavelengths, pseudocolored and digitally merged. Note the presence of yellow/orange micron size patches when Bodipy-D-erythro-LacCer was used at high concentrations. No such domains were observed using Bodipy-L-threo-LacCer. b Mapping endocytic markers to PM domains. HSFs were co-incubated with $5 \mu \mathrm{M}$ Bodipy-D-erythro- or L-threo-LacCer and AF647-labeled anti-

microdomains by Simian Virus 40 binding or CTxB antibody cross-linking (Singh et al. 2007a). L-threo-C8-LacCer also inhibited $\beta 1$-integrin activation, Simian Virus 40 infection, and endocytosis via caveolae (Singh et al. 2007a). Based on these studies and the above mentioned work with fluorescent analogs of LacCer, we suggest that the L-threo stereoisomer of LacCer may perturb the formation of

$\beta 1$ integrin Fab. c Fluorescence emission spectra of small unilamellar vesicles formed from POPC and containing 1, 2, 5, or $10 \mathrm{~mol} \% \mathrm{Bo}-$ dipy-D-erythro-LacCer or -L-threo-LacCer. Note the presence of monomer $(515 \mathrm{~nm})$ and excimer $(620 \mathrm{~nm})$ fluorescence emission peaks when the D-erythro isomer was used at high mol \% fractions, similar to Bodipy-Cer in Fig. 1b. In contrast, no excimer fluorescence was seen under the same conditions using the L-threo isomer. $\mathbf{d}$ Molecular models of Bodipy-LacCer stereoisomers created using the Chem3D program (Ultra 9.0, CambridgeSoft Corp., Cambridge, MA, USa). All data are from (Singh et al. 2006), reproduced with permission

microdomains by interfering with the close packing of endogenous SLs.

\section{Other Bodipy-SLs that label the PM}

Our studies of other Bodipy-tagged SLs aside from LacCer suggest that some of these lipid analogs may be useful for 
A

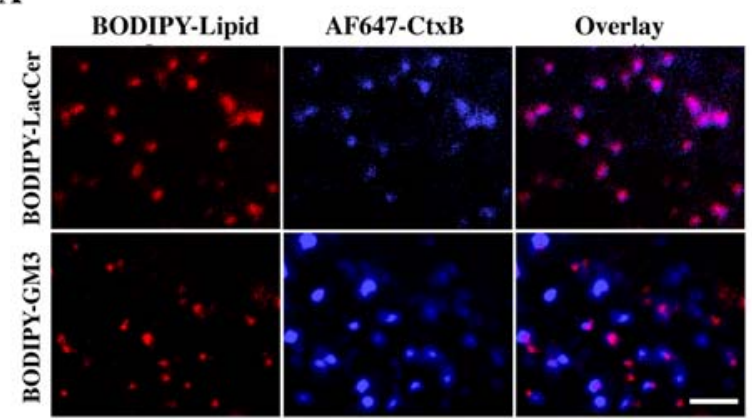

B

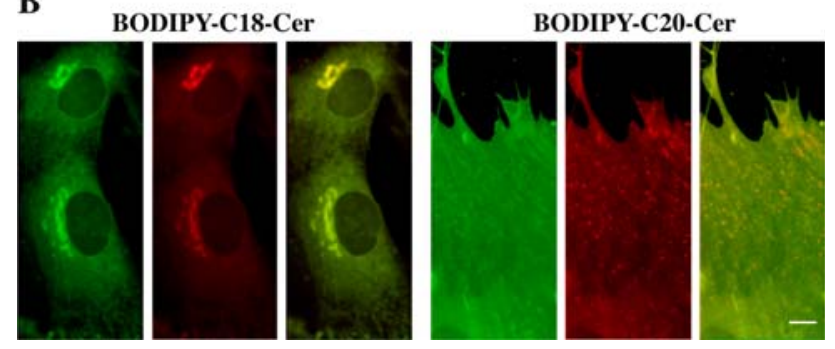

Fig. 5 Comparison of microdomains highlighted by Bodipy-LacCer vs Bodipy-GM $\mathrm{GM}_{3}$ ganglioside. a) Co-localization of Bodipy-D-erythroLacCer and-GM3 with $\beta 1$-integrin. Human skin fibroblasts were incubated $30 \mathrm{~min}$ at $10^{\circ} \mathrm{C}$ with $5 \mu \mathrm{M}$ Bodipy-lipid and AF647-Fab fragment directed against $\beta 1$-integrin. Bodipy-lipid was visualized by its red emission and $\beta 1$-integrin Fab using a far red filter. Note the colocalization of Bodipy-LacCer but not GM3 with $\beta 1$-integrin Fab. b Comparison of human skin fibroblast labeling with Bodipy-C18 (sphingosine)-Cer and C20(sphingosine)-Cer. Cells were incubated for $30 \mathrm{~min}$ at $10^{\circ} \mathrm{C}$ and then immediately imaged under the microscope at $10^{\circ} \mathrm{C}$. Note the extensive PM labeling seen with the $\mathrm{C} 20$ analog and greater labeling of the Golgi apparatus with the $\mathrm{C} 18 \mathrm{Cer}$

the study of PM microdomains whereas others may not be appropriate probes. In unpublished studies, we compared the microdomains induced by Bodipy-D-erythro- $\mathrm{GM}_{3}$ ganglioside with those of Bodipy-D-erythro-LacCer. Although areas of concentrated lipid analog as indicated by red fluorescence were visualized on the PM with Bodipy-Derythro- $\mathrm{GM}_{3}$, these structures did not co-localize with CTxB (Fig. 5a, b) or $\beta 1$-integrin (not shown). Thus, Bodipy-D-erythro- $\mathrm{GM}_{3}$ appears to highlight microdomains that are different from those seen with Bodipy-D-erythroLacCer. This concept is consistent with previous observations that endogenous $\mathrm{GM}_{3}$ exists in different microdomains from domains enriched in $\mathrm{GM}_{1}$ ganglioside and caveolin (Fujita et al. 2007; Iwabuchi et al. 1998; Janich and Corbeil 2007).

Several Bodipy-SLs have been found to undergo endocytosis by the same or similar mechanisms to BodipyLacCer (e.g., via caveolae) in rat fibroblasts (Singh et al. 2003). These lipids include Bodipy-derivatives of maltosyl (Glc-Glc)-Cer, triosyl (Gal-Gal-Glc)-ceramide (globoside), sulfatide, and $\mathrm{GM}_{1}$ ganglioside (see Fig. 2a). While these lipid analogs have not been investigated for their ability to partition into microdomains on the PM, their endocytosis via the same mechanism suggests that these lipids may organize into similar domains prior to internalization.

We have also investigated the internalization mechanism of the Bodipy-SM stereoisomers. We found that Bodipy-Derythro-SM is internalized via a nystatin (inhibits endocytosis via caveolae)-sensitive, chlorpromazine (inhibits clathrin-dependent endocytosis)-insensitive mechanism in human skin fibroblasts, similar to the behavior of BodipyD-erythro-LacCer. However, Bodipy-L-threo-SM endocytosis is more sensitive to chlorpromazine, and is similar to the results found with Bodipy-L-threo-LacCer (Singh et al. 2006). Thus, we speculate that the Bodipy-SM stereoisomers will partition into PM domains similarly to their LacCer counterparts. However, some SM analogs are rapidly hydrolyzed to Cer in several cell types, even at low temperature (Koval and Pagano 1989, 1990). Thus, in microscopy studies utilizing fluorescent SM analogs, it is important to analyze the metabolism of SM (e.g. by lipid extraction and TLC) to ensure that the fluorescence intensity reflects that of SM and not of Cer or other metabolites (see below). A possible solution to this problem would be to prepare a nonhydrolyzable analog of SM; other such SLs have been synthesized (Schwarzmann et al. 1995).

Finally, we have found that some Bodipy-SLs are inappropriate for studies of cell surface microdomains because they are internalized via non-vesicular mechanisms (i.e., they "flip" by active or passive transport). Bodipy-Cer with the typical C18 long-chain base has been shown by us to be internalized by cells even a low temperature (e.g., $\left.10^{\circ} \mathrm{C}\right)$ where endocytosis is not supported [see (Pagano et al. 1991) and Fig. 5b)]. In unpublished studies, we compared the behavior of Bodipy-Cer with different sphingosine chain lengths (C12, C14, C16, C18, and C20). Of these, all of the shorter chain length Bodipy-Cer analogs were internalized rapidly at low temperature; however, Bodipy-Cer with a $\mathrm{C} 20$ long-chain base labeled the $\mathrm{PM}$ at $10^{\circ} \mathrm{C}$ (Fig. 5b), suggesting that it could be used as a probe for monitoring the formation of Cer-enriched microdomains at the PM. Bodipy-GlcCer is also internalized at least in part by a non-vesicular mechanism that does not require ATP but appears to be saturable, suggesting transport by a "flippase" (Martin and Pagano 1994). Because of their ability to enter the cell by non-endocytic processes Bodipy-Cer (C18) and Bodipy-GlcCer in general are not useful for studies requiring prolonged labeling of the PM, particularly at physiological temperatures.

\section{Bodipy-cholesterol}

Free cholesterol is thought to be transported through the cell by multiple mechanisms including transbilayer 
diffusion and active transport as well as within vesicular structures (Fielding and Fielding 1997; Ikonen 2008; Prinz 2002; Schroeder et al. 2001). When cells are incubated with Bodipy-cholesterol, prominent PM labeling is observed at short time points (2-5 min); however, upon longer incubations ( $\geq 30 \mathrm{~min}$ ), fluorescence labeling of intracellular structures is also observed (Hölttä-Vuori et al. 2008). Thus, the use Bodipy-cholesterol for detection of PM microdomains remains to be explored.

\section{Labeling of microdomains on membranes of intracellular organelles}

In addition to the occurrence of microdomains at the PM, laterally segregated microdomains are thought to occur on intracellular membranes. Indeed, early papers by Simons and colleagues identified detergent-insoluble complexes of lipids and proteins in Golgi-derived vesicles destined for the apical membrane of MDCK cells (Fiedler et al. 1993; Kurzchalia et al. 1992). These observations suggested that certain SL-enriched membrane regions were segregated from others in the Golgi and were critical in the development of the "lipid raft" hypothesis. Microdomains are also expected to be found on early and recycling endosomes. Both of these organelles have been shown to be enriched in SLs and cholesterol (Evans and Hardison 1985; Gagescu et al. 2000; Hornick et al. 1997). Separate domains on early and recycling endosomes also have been visualized using fluorescently tagged Rab proteins (Rabs 4, 5, and 11) which can highlight distinct regions on the same endosome (Sonnichsen et al. 2000). Studies employing immuno-electron microscopy have shown that some internalized ligand-receptor complexes become separated from each other in early endosomes, with ligands (e.g., asialoglycoprotein, low-density lipoprotein) being concentrated in the endosomal lumen and their receptors enriched in tubular structures associated with the endosomes [(Geuze et al. 1983, 1987); see model in Fig. 6a]. Receptors in these tubules can recycle to the PM either directly (rab4-dependent or "fast recycling") or via passage through the recycling endosome (rab11-dependent or "slow recycling"). The transferrin receptor (TfR) has a somewhat unique itinerary in that after endocytosis and delivery to the early endosome Tfr does not dissociate from transferrin (Tfn) but rather both Tfn and TfR move into tubules emanating from the early endosome. A portion of the TfR/Tfn complex is then transported directly back to the PM but most of the ligand/receptor complex is transported to the recycling endosome and then returned to the $\mathrm{PM}$.
Visualization of endosomal microdomains with Bodipy-LacCer

After endocytosis via caveolae Bodipy-LacCer is transported through early endosomes (Sharma et al. 2003). By allowing Bodipy-LacCer endocytosis $(2.5 \mu \mathrm{M})$ to occur for $1 \mathrm{~h}$ at $16^{\circ} \mathrm{C}$, most of the endocytosed lipid is retained in the early endosome (Sharma et al. 2003). Under these conditions, Bodipy-LacCer can be seen in endosomes in the red and green fluorescence channels (Fig. 6b). In some cases, both red and green fluorescence is associated with the same endosomal structure but segregated into different regions of the membrane, suggestive of microdomains [Fig. 6b; (Sharma et al. 2003)]. When the same experiment is performed with AlexaFluor 647-labeled Tfn (AF647-Tfn) added together with Bodipy-LacCer, the AF647-Tfn (visualized with a far red filter) is associated more closely with the red (concentrated) Bodipy-LacCer regions than with the green Bodipy-fluorescence [Fig. 6c (Sharma et al. 2003)]. On warming to $37^{\circ} \mathrm{C}$ for $5 \mathrm{~min}$, the AF647-Tfn and Bodipy-red fluorescence become separated from the green Bodipy-LacCer fluorescence (Fig. 6d). Given the known itinerary of Tfn through early endosomes, and then recycling either directly or via recycling endosomes, these results are interpreted as showing that a concentrated pool of Bodipy-LacCer buds off from the early endosome with Tfn to be recycled to the PM (Sharma et al. 2003). We have provided further evidence of Bodipy-LacCer recycling in other studies (Choudhury et al. 2004). Other Bodipy-SLs that are internalized by endocytosis (see above) are expected to behave similarly but have not been examined for their utility in visualizing endosomal microdomains.

Visualization of Golgi microdomains with Bodipy-LacCer

As mentioned above, the segregation of protein and lipid cargo into microdomains at the Golgi membrane may be a mechanism for the delivery of different classes of materials to different destinations. For example, it has been proposed that some proteins destined for delivery to apical membranes of epithelial cells are segregated at the Golgi from proteins that will be delivered to basolateral membranes (Simons and van Meer 1988). In addition, just as there are multiple types of budding from the PM for endocytosis [e.g. clathrin-dependent vs clathrin-independent mechanisms (Mayor and Pagano 2007)], there are multiple mechanisms by which vesicles or tubules are proposed to exit the Golgi complex (Holthuis et al. 2001; Kepes et al. 2005).

Several Bodipy-SLs have been shown to become concentrated in the Golgi apparatus when added to multiple cell types. These include Bodipy-Cer, -D-erythro-LacCer, -Lthreo-LacCer, -SM, and -GlcCer [unpublished observations and (Chen et al. 1997; Choudhury et al. 2002b; Martin and 

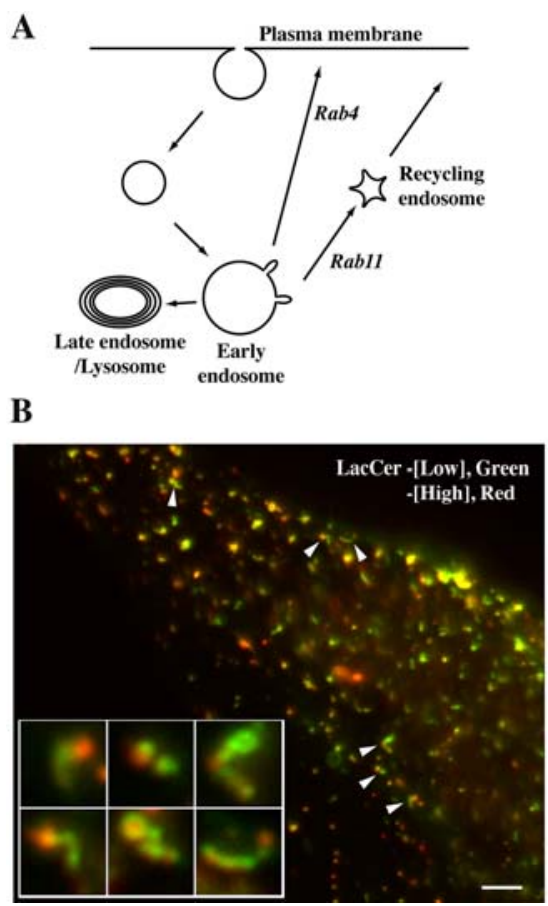

Fig. 6 Use of Bodipy-lipids to label intracellular microdomains. a Model of endosomal trafficking showing transport from the PM, through early and recycling endosomes, followed by recycling back to the PM. In some cases cargo or receptors may be transported from early endosomes to late endosomes and lysosomes for degradation. b Human skin fibroblasts were incubated with Bodipy-D-erythro-LacCer for $60 \mathrm{~min}$ at $16^{\circ} \mathrm{C}$ to load the early endosomal compartment. Cells then were subject to back exchange to remove PM fluorescence and imaged at green and red wavelengths. Note that green and red fluorescence was sometimes found on distinct regions of individual endosomes. In c, d human skin fibroblasts were incubated with Bodipy-D-erythroLacCer and AF647-Tfn for 60 min at $16^{\circ} \mathrm{C}$ to load the early endosomal compartment as in panel (a). Cells were then subjected to back

Pagano 1994; Pagano et al. 1989; Puri et al. 2001; Sillence et al. 2002)]. Bodipy-Cer has been shown to localize mainly in the trans-Golgi and trans-Golgi network using electron microscopy and a photoconversion method that is based on the photobleaching of the Bodipy-fluorophore in the presence of diaminobenzidine (Ladinsky et al. 1994; Pagano et al. 1991). It is unknown if other Bodipy-SLs are incorporated into the same Golgi compartment or other cisternae. When viewed by fluorescence microscopy, each of these BodipySLs can be seen at the Golgi apparatus at both green and red wavelengths (e.g., Fig. 1d). With the advent of high resolution fluorescence methods, it may be possible in the future to detect SL domains at the Golgi using these analogs. One feature of Bodipy-Cer may be of further interest in this regard. As Bodipy-Cer is rapidly metabolized (mainly to Bodipy$\mathrm{SM}$ ) in the Golgi appparatus, it may be possible to load cells with Bodipy-Cer, allow its conversion to SM in the Golgi apparatus, and then view the budding of vesicles from the Golgi which are enriched in Bodipy-SM. Early data from (Pagano et al. 1991) suggests the feasibility of this approach.
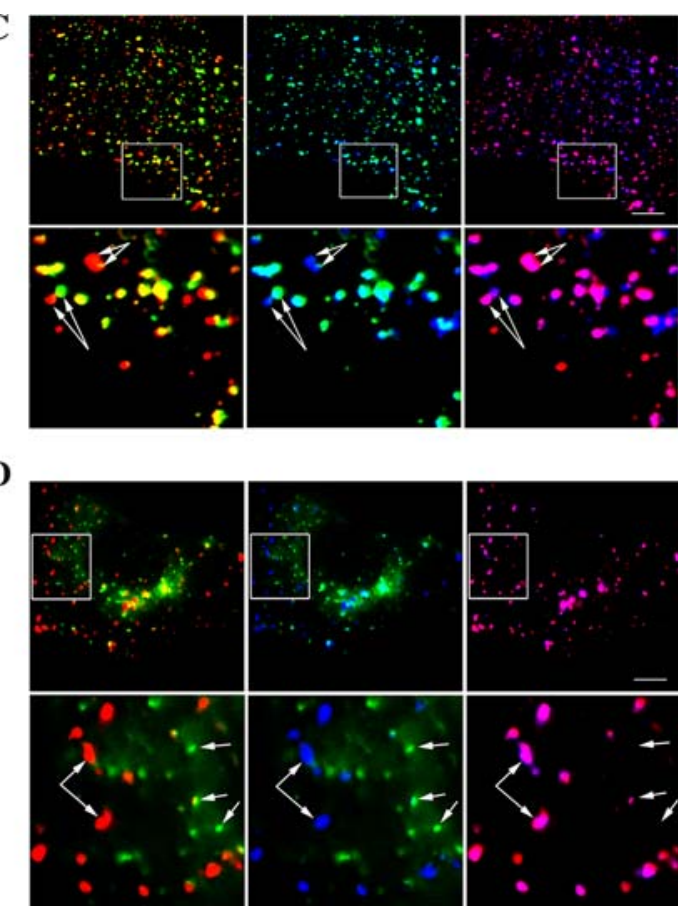

exchange and acid stripping to remove PM fluorescence. Cells were then incubated at $37^{\circ} \mathrm{C}$ for $\mathbf{c} 0$ or $\mathbf{d ~} 5 \mathrm{~min}$, and photographed at green and red wavelengths (for low and high concentrations of Bodipy) and at far red wavelengths for Tfn. Images were rendered in pseudocolor with green and red corresponding to LacCer microdomains and blue corresponding to Tfn fluorescence. Note the close correspondence of red and blue in (c), indicating the presence of high concentrations of Bodipy-LacCer and Tfn in the same domains of endosomes. Upon warming to $37^{\circ} \mathrm{C}$ (panel d), the concentrated (red) pool of Bodipy-LacCer and Tfn become separated from the lower concentration (green) pool of Bodipy-LacCer, en route to recycling back to the PM. Panels (b-d) are reproduced from (Sharma et al. 2003), with permission

Visualization of intracellular membrane microdomains with Bodipy-cholesterol

Few studies have been performed so far with Bodipy-cholesterol; however, it has been shown that Bodipy-cholesterol is rapidly concentrated in endosomes in CHO cells (Hölttä-Vuori et al. 2008). In unpublished studies, we have also observed that Bodipy-cholesterol is rapidly transported to the intracellular structures in several other cell types including rat oligodendrocytes. Thus, Bodipy-cholesterol may be useful for visualizing cholesterol-enriched microdomains on intracellular membranes.

\section{Future applications and perspectives}

Although Bodipy-SLs have been used for cell biological research for many years, their application to the study of membrane microdomains has been recent. We have only begun to learn about the characteristics of PM domains 
highlighted by Bodipy-LacCer and how they are regulated. Our preliminary studies showing that Bodipy- $\mathrm{GM}_{3}$ is localized in different microdomains at the PM suggest the possibility that other Bodipy-derivatives of GSLs [e.g., GD or GT series gangliosides; for a review, see (Kolter et al. 2002)] may partition into distinct membrane domains. The labeling of intracellular organelles with Bodipy-cholesterol suggests the potential for using this lipid probe to investigate intracellular microdomains and the rapid transport of cholesterol into and out of these microdomains.

Thus far our studies of PM microdomains have relied on the use of low temperature $\left(4-10^{\circ} \mathrm{C}\right)$ to prevent endocytosis of Bodipy-SLs and thus allow the visualization of the lipid probes exclusively at the PM. It will be important to study the behavior of Bodipy-SL-labeled microdomains at physiological temperature $\left(37^{\circ} \mathrm{C}\right)$ to determine if they are similar to those seen at low temperature and to study their dynamics and stability. This kind of experiment may be executed by inhibiting the endocytosis of the lipid probe [e.g., for Bodipy-LacCer with a src kinase inhibitor or a dominant negative dynamin (Cheng et al. 2006; Puri et al. 2001; Sharma et al. 2004; Singh et al. 2003)].

Studies need to be performed to determine if some of the Bodipy-related fluorophores (e.g., Bodipy-TR) will be as useful as Bodipy-FL for labeling lipid probes. For example, would Bodipy-TR-SL readily transfer to cells from a BSA complex, would the Bodipy-TR fatty acid moiety associate with the PM, and would such probes faithfully mimic natural SLs (e.g., are they metabolized and/or transported to the Golgi apparatus)? Probes with red-shifted fluorescence would be very useful for co-localization with green fluorescent proteins, an approach that is not usually feasible with Bodipy-FL probes because their fluorescence overlaps with green fluorescent proteins. Another potential solution to the problem of Bodipy-FL fluorescence overlap with fluorescent proteins is the use of far-red shifted fluorescent protein tags, which are now commercially available. The ability to simultaneously visualize both a fluorescent SL and a microdomain-associated protein (e.g., Cav1/far red fluorescent protein) at the PM could provide new information about the coalescence of microdomains in the moments before endocytosis.

Finally, most of the Bodipy-SLs that have been discussed label the outer leaflet of the PM. Little information is available about the lipid composition or microdomain organization on the inner leaflet "beneath" the outer leaflet microdomains. The possibility of mapping outer leaflet Bodipy-SL microdomains to inner leaflet regions enriched in various phosphoinositides through the use of phosphatidylinositol-sensing domains or fluorescent proteins that target to inner leaflet microdomains (Abankwa et al. 2007; Lasserre et al. 2008) could provide new advances in our understanding of transbilayer coupling.
Acknowledgments Supported by National Institutes of Health grants GM-2242 and GM-60934 to REP and HL-083187 to RB. The authors thank Drs T. Kaptzan, D. Sharma, and R. Singh for providing unpublished data.

\section{References}

Abankwa D, Gorfe AA, Hancock JF (2007) Ras nanoclusters: molecular structure and assembly. Semin Cell Dev Biol 18:599-607

Anderson RG, Jacobson K (2002) A role for lipid shells in targeting proteins to caveolae, rafts, and other lipid domains. Science 296:1821-1825

Ariga T, McDonald MP, Yu RK (2008) Role of ganglioside metabolism in the pathogenesis of Alzheimer's disease-a review. J Lipid Res 49:1157-1175

Bittman R (2008) Synthetic sphingolipids as bioactive molecules: roles in regulation of cell function. In: Tadhg P. Begley (ed) Wiley encyclopedia of chemical biology. Wiley, New York. http:// mrw.interscience.wiley.com/emrw/9780470048672/home/

Chattopadhyay A, London E (1987) Parallax method for direct measurement of membrane penetration depth utilizing fluorescence quenching by spin-labeled phospholipids. Biochemistry 26:39-45

Chen CS, Martin OC, Pagano RE (1997) Changes in the spectral properties of a plasma membrane lipid analog during the first seconds of endocytosis in living cells. Biophys J 72:37-50

Cheng ZJ, Singh RD, Sharma DK, Holicky EL, Hanada K, Marks DL, Pagano RE (2006) Distinct mechanisms of clathrin-independent endocytosis have unique sphingolipid requirements. Mol Biol Cell 17:3197-3210

Choudhury A, Dominguez M, Puri V, Sharma DK, Narita K, Wheatley CW, Marks DL, Pagano RE (2002a) Rab proteins mediate Golgi transport of caveola-internalized glycosphingolipids and correct lipid trafficking in Niemann-Pick C cells. J Clin Invest 109:15411550

Choudhury A, Sharma DK, Wheatley CL, Marks DL, Pagano RE (2002b) Abnormal sphingolipid recycling in Niemann Pick-A (NP-A) fibroblasts. Mol Biol Cell 13S:93a

Choudhury A, Sharma DK, Marks DL, Pagano RE (2004) Elevated endosomal cholesterol levels in Niemann-Pick cells inhibit Rab4 and perturb membrane recycling. Mol Biol Cell 15:4500-4511

Devaux PF, Morris R (2004) Transmembrane asymmetry and lateral domains in biological membranes. Traffic 5:241-246

Edidin M (2003) The state of lipids rafts: from model membranes to cells. Annu Rev Biophys Biomol Struct 32:257-283

Emura C, Higuchi R, Miyamoto T, Van Soest RW (2005) Amphimelibiosides A-F, six new ceramide dihexosides isolated from a Japanese marine sponge Amphimedon sp. J Org Chem 70:3031-3038

Evans WH, Hardison WG (1985) Phospholipid, cholesterol, polypeptide and glycoprotein composition of hepatic endosome subfractions. Biochem J 232:33-36

Fahy E, Subramaniam S, Brown HA, Glass CK, Merrill AH Jr, Murphy RC, Raetz CR, Russell DW, Seyama Y, Shaw W, Shimizu T, Spener F, van Meer G, VanNieuwenhze MS, White SH, Witztum JL, Dennis EA (2005) A comprehensive classification system for lipids. J Lipid Res 46:839-861

Fiedler K, Kobayashi T, Kurzchalia TV, Simons K (1993) Glycosphingolipid-enriched, detergent-insoluble complexes in protein sorting in epithelial cells. Biochemistry 32:6365-6373

Field H, Sherwin T, Smith AC, Gull K, Field MC (2000) Cell-cycle and developmental regulation of TbRAB31 localisation, a GTPlocked Rab protein from Trypanosoma brucei. Mol Biochem Parasitol 106:21-35

Fielding CJ, Fielding PE (1997) Intracellular cholesterol transport. J Lipid Res 38:1503-1521 
Fujita A, Cheng J, Hirakawa M, Furukawa K, Kusunoki S, Fujimoto T (2007) Gangliosides GM1 and GM3 in the living cell membrane form clusters susceptible to cholesterol depletion and chilling. Mol Biol Cell 18:2112-2122

Gagescu R, Demaurex N, Parton RG, Hunziker W, Huber LA, Gruenberg J (2000) The recycling endosome of Madin-Darby canine kidney cells is a mildly acidic compartment rich in raft components. Mol Biol Cell 11:2775-2791

Gangalum RK, Schibler MJ, Bhat SP (2004) Small heat shock protein alpha B-crystallin is part of cell cycle-dependent Golgi reorganization. J Biol Chem 279:43374-43377

Gao JM, Zhang AL, Chen H, Liu JK (2004) Molecular species of ceramides from the ascomycete truffle Tuber indicum. Chem Phys Lipids 131:205-213

Geuze HJ, Slot JW, Strous GJ, Lodish HF, Schwartz AL (1983) Intracellular site of asialoglycoprotein receptor-ligand uncoupling: double-label immunoelectron microscopy during receptor-mediated endocytosis. Cell 32:277-287

Geuze HJ, Slot JW, Schwartz AL (1987) Membranes of sorting organelles display lateral heterogeneity in receptor distribution. J Cell Biol 104:1715-1723

Hakomori S (2003) Structure, organization, and function of glycosphingolipids in membrane. Curr Opin Hematol 10:16-24

Hannun YA, Obeid LM (2008) Principles of bioactive lipid signalling: lessons from sphingolipids. Nat Rev Mol Cell Biol 9:139-150

Harder T, Engelhardt KR (2004) Membrane domains in lymphocytes-from lipid rafts to protein scaffolds. Traffic 5:265-275

Harder T, Scheiffele P, Verkade P, Simons K (1998) Lipid domain structure of the plasma membrane revealed by patching of membrane components. J Cell Biol 141:929-942

Harding A, Hancock JF (2008) Ras nanoclusters: combining digital and analog signaling. Cell Cycle 7:127-134

Holland WL, Summers SA (2008) Sphingolipids, insulin resistance, and metabolic disease: new insights from in vivo manipulation of sphingolipid metabolism. Endocr Rev 29:381-402

Holthuis JC, Pomorski T, Raggers RJ, Sprong H, Van Meer G (2001) The organizing potential of sphingolipids in intracellular membrane transport. Physiol Rev 81:1689-1723

Hölttä-Vuori M, Uronen RL, Repakova J, Salonen E, Vattulainen I, Panula P, Li Z, Bittman R, Ikonen E (2008) BODIPY-cholesterol: a new tool to visualize sterol trafficking in living cells and organisms. Traffic. doi:10.1111/j.1600-0854.2008.00801.x

Hornick CA, Hui DY, DeLamatre JG (1997) A role for retrosomes in intracellular cholesterol transport from endosomes to the plasma membrane. Am J Physiol 273:C1075-C1081

Ikonen E (2008) Cellular cholesterol trafficking and compartmentalization. Nat Rev Mol Cell Biol 9:125-138

Inagaki M, Ikeda Y, Kawatake S, Nakamura K, Tanaka M, Misawa E, Yamada M, Higuchi R (2006) Isolation and structure of four new ceramides from the starfish Luidia maculata. Chem Pharm Bull (Tokyo) 54:1647-1649

Ishii T, Okino T, Mino Y (2006) A ceramide and cerebroside from the starfish asterias amurensis Lutken and their plant-growth promotion activities. J Nat Prod 69:1080-1082

Iwabuchi K, Handa K, Hakomori S (1998) Separation of "glycosphingolipid signaling domain" from caveolin-containing membrane fraction in mouse melanoma B 16 cells and its role in cell adhesion coupled with signaling. J Biol Chem 273:33766-33773

Jacobson K, Mouritsen OG, Anderson RG (2007) Lipid rafts: at a crossroad between cell biology and physics. Nat Cell Biol 9:7-14

Janich P, Corbeil D (2007) GM1 and GM3 gangliosides highlight distinct lipid microdomains within the apical domain of epithelial cells. FEBS Lett 581:1783-1787

Kaiser RD, London E (1998) Determination of the depth of BODIPY probes in model membranes by parallax analysis of fluorescence quenching. Biochim Biophys Acta 1375:13-22
Kepes F, Rambourg A, Satiat-Jeunemaitre B (2005) Morphodynamics of the secretory pathway. Int Rev Cytol 242:55-120

Kirkham M, Parton RG (2005) Clathrin-independent endocytosis: new insights into caveolae and non-caveolar lipid raft carriers. Biochim Biophys Acta 1746:350-363

Kolter T, Proia RL, Sandhoff K (2002) Combinatorial ganglioside biosynthesis. J Biol Chem 277:25859-25862

Koval M, Pagano RE (1989) Lipid recycling between the plasma membrane and intracellular compartments: transport and metabolism of fluorescent sphingomyelin analogs in cultured fibroblasts. J Cell Biol 108:2169-2181

Koval M, Pagano RE (1990) Sorting of an internalized plasma membrane lipid between recycling and degradative pathways in normal and Niemann-Pick, type A fibroblasts. J Cell Biol 111:429-442

Kurzchalia TV, Dupree P, Parton RG, Kellner R, Virta H, Lehnert M, Simons K (1992) VIP21, a 21-kD membrane protein is an integral component of trans-Golgi-network-derived transport vesicles. J Cell Biol 118:1003-1014

Kusumi A, Suzuki K (2005) Toward understanding the dynamics of membrane-raft-based molecular interactions. Biochim Biophys Acta 1746:234-251

Ladinsky M, Kremer J, Furchinitti P, McIntosh J, Howell K (1994) HVEM tomography of the trans-Golgi network: structural insights and identification of a lace-like vesicle coat. J Cell Biol 127:29-38

Lasserre R, Guo XJ, Conchonaud F, Hamon Y, Hawchar O, Bernard AM, Soudja SM, Lenne PF, Rigneault H, Olive D, Bismuth G, Nunes JA, Payrastre B, Marguet D, He HT (2008) Raft nanodomains contribute to Akt/PKB plasma membrane recruitment and activation. Nat Chem Biol 4:538-547

Lawrence JC, Saslowsky DE, Edwardson JM, Henderson RM (2003) Real-time analysis of the effects of cholesterol on lipid raft behavior using atomic force microscopy. Biophys J 84:1827-1832

Li Z, Bittman R (2007) Synthesis and spectral properties of cholesterol- and FTY720-containing boron dipyrromethene dyes. J Org Chem 72:8376-8382

Li XA, Everson WV, Smart EJ (2005) Caveolae, lipid rafts, and vascular disease. Trends Cardiovasc Med 15:92-96

Li Z, Mintzer E, Bittman R (2006) First synthesis of free cholesterolBODIPY conjugates. J Org Chem 71:1718-1721

Ling T, Xia T, Wan X, Li D, Wei X (2006) Cerebrosides from the roots of Serratula chinensis. Molecules 11:677-683

London E, Brown DA (2000) Insolubility of lipids in triton X-100: physical origin and relationship to sphingolipid/cholesterol membrane domains (rafts). Biochim Biophys Acta 1508:182-195

Marguet D, Lenne PF, Rigneault H, He HT (2006) Dynamics in the plasma membrane: how to combine fluidity and order. EMBO J 25:3446-3457

Marks DL, Singh RD, Choudhury A, Wheatley CL, Pagano RE (2005) Use of fluorescent sphingolipid analogs to study lipid transport along the endocytic pathway. Methods 36:186-195

Martin OC, Pagano RE (1987) Transbilayer movement of fluorescent analogs of phosphatidylserine and phosphatidylethanolamine at the plasma membrane of cultured cells: evidence for a proteinmediated and ATP-dependent process(es). J Biol Chem 262:5890-5898

Martin OC, Pagano RE (1994) Internalization and sorting of a fluorescent analog of glucosylceramide to the Golgi apparatus of human skin fibroblasts: utilization of endocytic and nonendocytic transport mechanisms. J Cell Biol 125:769-781

Maxfield FR, Tabas I (2005) Role of cholesterol and lipid organization in disease. Nature 438:612-621

Mayor S, Pagano RE (2007) Pathways of clathrin-independent endocytosis. Nat Rev Mol Cell Biol 8:603-612

Mishra S, Joshi PG (2007) Lipid raft heterogeneity: an enigma. J Neurochem 103(Suppl 1):135-142 
Oueslati MH, Mighri Z, Ben Jannet H, Abreu PM (2005) New ceramides from Rantherium suaveolens. Lipids 40:1075-1079

Pagano RE, Sepanski MA, Martin OC (1989) Molecular trapping of a fluorescent ceramide analog at the Golgi apparatus of fixed cells: interaction with endogenous lipids provides a trans-Golgi marker for both light and electron microscopy. J Cell Biol 109:2067-2079

Pagano RE, Martin OC, Kang HC, Haugland RP (1991) A novel fluorescent ceramide analogue for studying membrane traffic in animal cells: accumulation at the Golgi apparatus results in altered spectral properties of the sphingolipid precursor. J Cell Biol 113:1267-1279

Parton RG, Hancock JF (2004) Lipida rafts and plasma membrane microorganization: insights from Ras. Trends Cell Biol 14:141-147

Pike LJ (2006) Rafts defined: a report on the Keystone symposium on lipid rafts and cell function. J Lipid Res 47:1597-1598

Prinz W (2002) Cholesterol trafficking in the secretory and endocytic systems. Semin Cell Dev Biol 13:197-203

Puri V, Watanabe R, Dominguez M, Sun X, Wheatley CL, Marks DL, Pagano RE (1999) Cholesterol modulates membrane traffic along the endocytic pathway in sphingolipid storage diseases. Nat Cell Biol 1:386-388

Puri V, Watanabe R, Singh RD, Dominguez M, Brown JC, Wheatley CL, Marks DL, Pagano RE (2001) Clathrin-dependent and -independent internalization of plasma membrane sphingolipids initiates two Golgi targeting pathways. J Cell Biol 154:535-547

Qin W, Rohand T, Dehaen W, Clifford JN, Driesen K, Beljonne D, Van Averbeke B, Van der Auweraer M, Boens N (2007) Boron dipyrromethene analogs with phenyl, styryl, and ethynylphenyl substituents: synthesis, photophysics, electrochemistry, and quantum-chemical calculations. J Phys Chem A 111:8588-8597

Rajendran L, Simons K (2005) Lipid rafts and membrane dynamics. J Cell Sci 118:1099-1102

Regina Todeschini A, Hakomori SI (2008) Functional role of glycosphingolipids and gangliosides in control of cell adhesion, motility, and growth, through glycosynaptic microdomains. Biochim Biophys Acta 1780:421-433

Schnitzer JE, McIntosh DP, Dvorak AM, Liu J, Oh P (1995) Separation of caveolae from associated microdomains of GPI-anchored proteins. Science 269:1435-1439

Schroeder F, Gallegos AM, Atshaves BP, Storey SM, McIntosh AL, Petrescu AD, Huang H, Starodub O, Chao H, Yang H, Frolov A, Kier AB (2001) Recent advances in membrane microdomains: rafts, caveolae, and intracellular cholesterol trafficking. Exp Biol Med (Maywood) 226:873-890

Schwarzmann G, Hofmann P, Pütz U, Albrecht B (1995) Demonstration of direct glycosylation of nondegradable gycosylceramide analogs in cultured cells. J Biol Chem 270:21271-21276

Sharma DK, Choudhury A, Singh RD, Wheatley CL, Marks DL, Pagano RE (2003) Glycosphingolipids internalized via caveolar-related endocytosis rapidly merge with the clathrin pathway in early endosomes and form microdomains for recycling. J Biol Chem 278:7564-7572

Sharma DK, Brown JC, Choudhury A, Peterson TE, Holicky E, Marks DL, Simari R, Parton RG, Pagano RE (2004) Selective stimula- tion of caveolar endocytosis by glycosphingolipids and cholesterol. Mol Biol Cell 15:3114-3122

Sharma DK, Brown JC, Cheng Z, Holicky EL, Marks DL, Pagano RE (2005) The glycosphingolipid, lactosylceramide, regulates beta1integrin clustering and endocytosis. Cancer Res 65:8233-8241

Shaw JE, Epand RF, Epand RM, Li Z, Bittman R, Yip CM (2006) Correlated fluorescence-atomic force microscopy of membrane domains: structure of fluorescence probes determines lipid localization. Biophys J 90:2170-2178

Shayman JA, Radin NS (1991) Structure and function of renal glycosphingolipids. Am J Physiol 260:F291-F302

Sillence DJ, Puri V, Marks DL, Butters TD, Dwek RA, Pagano RE, Platt FM (2002) Glucosylceramide modulates membrane traffic along the endocytic pathway. J Lipid Res 43:1837-1845

Simons K, Ehehalt R (2002) Cholesterol, lipid rafts, and disease. J Clin Invest 110:597-603

Simons K, Ikonen E (1997) Functional rafts in cell membranes. Nature 387:569-572

Simons K, Toomre D (2000) Lipid rafts and signal transduction. Nat Rev Mol Cell Biol 1:31-39

Simons K, van Meer G (1988) Lipid sorting in epithelial cells. Biochemistry 27:6197-6202

Singh RD, Puri V, Valiyaveettil JT, Marks DL, Bittman R, Pagano RE (2003) Selective caveolin-1-dependent endocytosis of glycosphingolipids. Mol Biol Cell 14:3254-3265

Singh RD, Liu Y, Wheatley CL, Holicky EL, Makino A, Marks DL, Kobayashi T, Subramaniam G, Bittman R, Pagano RE (2006) Caveolar endocytosis and microdomain association of a glycosphingolipid analog is dependent on its sphingosine stereochemistry. J Biol Chem 281:30660-30668

Singh RD, Holicky EL, Cheng Z, Kim SY, Wheatley CL, Marks DL, Bittman R, Pagano RE (2007a) Inhibition of caveolar uptake, SV40 infection, and $\beta 1$-integrin signaling by a non-natural glycosphingolipid stereoisomer. J Cell Biol 176:895-901

Singh RD, Marks DL, Pagano RE (2007b) The use of lipid analogs to dissect lipid trafficking. Curr Protoc Cell Biol Suppl 35:24.1.124.1.19

Smart EJ, Ying YS, Mineo C, Anderson RG (1995) A detergent-free method for purifying caveolae membrane from tissue culture cells. Proc Natl Acad Sci USA 92:10104-10108

Sonnichsen B, De Renzis S, Nielsen E, Rietdorf J, Zerial M (2000) Distinct membrane domains on endosomes in the recycling pathway visualized by multicolor imaging of Rab4, Rab5, and Rab11. J Cell Biol 149:901-914

Watanabe R, Asakura K, Rodriguez M, Pagano RE (1999) Internalization and sorting of plasma membrane sphingolipid analogues in differentiating oligodendrocytes. J Neurochem 73:1375-1383

Yamada K, Harada Y, Miyamoto T, Isobe R, Higuchi R (2000) Constituents of holothuroidea.9. Isolation and structure of a new ganglioside molecular species from the sea cucumber Holothuria pervicax. Chem Pharm Bull (Tokyo) 48:157-159

Zhang WK, Xu JK, Zhang XQ, Yao XS, Ye WC (2007) Sphingolipids with neuritogenic activity from Euphorbia sororia. Chem Phys Lipids 148:77-83 